\title{
Consolidating Fundamental and Exploiting Border: Structural Optimization of Teaching Ethics
}

\author{
Jia Yanqi \\ Faculty of Education, Beijing Normal University, Beijing, China
}

\section{Email address:}

1175165034@qq.com

\section{To cite this article:}

Jia Yanqi. Consolidating Fundamental and Exploiting Border: Structural Optimization of Teaching Ethics. Science Innovation. Vol. 6, No. 1, 2018, pp. 20-23. doi: 10.11648/j.si.20180601.15

Received: January 19, 2018; Accepted: January 30, 2018; Published: March 9, 2018

\begin{abstract}
Although the research of teaching ethics has made a certain achievement recently, there are also severe structural deficiencies. From the perspective of the participants in teaching activities that related to ethics, the defect manifests as paying close attention to the instructing ethics of teachers, but forgetting the learning ethics of students. While from the research perspective of teaching ethics, the defect presents as emphasizing normative ethics which based on the power of stipulations, but losing the sight of other multiple ethical review. In order to address the above problems, it is necessary to take some strategical adjustment in the future research of teaching ethics, including repeating teaching concept, ranking and enhancing learning ethics among students, what's more, breaking through the single perspective of normative ethics, and enhancing the aptness of ethical value judgment.
\end{abstract}

Keywords: Teaching Ethics, Learning Ethics, Consolidating Fundamental and Exploiting Border, Caring Ethics

\section{固本拓边: 教学伦理研究的结构性优化}

\section{贾彦琪}

教育学部, 北京师范大学, 北京, 中国

\section{邮箱}

1175165034@qq.com

摘要: 当前教学伦理研究虽已取得了一定成就, 但仍存在较为明显的结构性缺失, 从教学伦理活动的参与主体来看, 表现为专注教师“教”的伦理研究, 遗忘学生“学”的伦理研究; 从教学伦理的研究视角来看, 体现在凸显以规约为基调 的规范伦理学, 冷落其他多元的伦理审视。为有效应对上述问题, 未来的教学伦理研究必须做出战略性调整, 如重申 教学内涵, 重视与加强学生的学习伦理研究; 冲破规范伦理的单一视角, 提升教学伦理价值选择的适切性。

关键词: 教学伦理, 学习伦理, 固本拓边, 结构性缺失

\section{1. 引言}

“教学是个多维存在, 除具科学性与艺术性外, 还具 有伦理性, 且伦理性之于教学具有首要性, 教学是立于科 学达于艺术之伦理性活动。”[1]“开展教学伦理研究既是教 学论研究应有之义, 又是回应与应对当前教学实践伦理缺
位之现实诉求, 可以说关注与重视教学伦理研究不仅必要 而且亟须, 要高度重视并积极开展教学伦理研究。”[2]可 喜的是, 近年来随着人们对教学伦理研究之重要与必要的 意识与重视, 在教学论领域掀起了一股教学伦理研究热, 并取得了一定成果。然遗憾的是, 由于人们对教学、伦理 以及教学伦理研究认知的偏差和片面, 当前教学伦理研究 之结构尚存在较大缺失。一方面, 就教学活动主体而言, 
当前的教学伦理研究偏指教师一方, 对于学生在伦理活动 中的地位、作用、规范和行为则少有论及。[3]另一方面, 就教学伦理研究视角而言, 仍然以规范伦理研究为主导思 路, 对于美德伦理、关怀伦理等相关理念的关注较为缺乏。 长此以往, 不仅将直接导致教学伦理要素的缺席和研究的 失衡, 同时亦会影响到教学实践的开展。有鉴于此, 笔者 在揭示当前教学伦理研究突出的结构性缺失基础上, 以 “固本拓边”的思路为统筹, 进一步就如何改进和完善当前 教学伦理研究提出了自己的一点思考与拙见, 以期对完善 教学伦理研究的结构体系有所助益, 并引发研究者对该问 题的进一步关注。

\section{2. 当前教学伦理研究的结构性缺失简析}

\section{1. 专注教师“教”的伦理, 遗忘学生“学”的伦理}

所谓教学, 顾名思义, 一定“永远包括教和学, 而且 不是简单地教加学。教和学是教学这同一事情的两个侧面, 是辩证统一的。”[4]从教学的本质出发, 教学伦理研究也 应该包含教师和学生这两个主体, 应以善恶为尺度规定师 生双方的行为规范, 但当前对于教学伦理的研究多着眼于 教师, 较少着眼于学生, 即便是关于学习方式中伦理问题 的探讨, 也大多归结到教师的教学设计, 而很少强调学生 自身的责任担当，[5]完全没有站在“师生共同体”的平台上, 有悖于课堂生活的真实形态。之所以出现关注教师“教”的 伦理, 遗忘学生“学”的伦理这一现象, 首先源于人们对于 师生关系的认识。一方面, 教师与学生相比, 拥有更多的 知识和经验, 在教学中居于主导地位, 这种不对等性关系 实际上就预示着教师应对教学伦理问题负有更大的责任。 另一方面, 教师还往往成为学生模仿的对象, 这更要求教 师必须身体力行, 率先垂范。正如有研究者指出的那样, 教师在教学中可能的不道德决策行为, 会使学生在意识和 行为上受到直接强化和替代强化, 无论学生能否明白教师 行为的性质, 也无论其被强化的过程是外显还是内隐。以 上两点都说明教师对学生所负责任重大, 尤其是在中小学 阶段, 因此, 人们在遇到教学伦理问题时便会不自觉地首 先想到教师, 遗忘和遮蔽了学生的伦理担当, 最终造成学 习伦理的研究缺失。其次, 人们对教师“教”的伦理的关注, 一定程度上也是研究惯性作用的结果。自从1985年《教学 伦理》出版到 20 世纪 90 年代, 人们对于教学伦理的探讨就 主要集中在教师身上, 认为教师是承担教学道德的主体。 我国的教学伦理研究也较多地集中于课堂教学中教师的 言行规范。20世纪30年代开始, 就有研究者涉足这一领域, 如自罗廷光在《教学通论》中提出了“教师品格”的概念, 建国后颁布了《中小学教师职业道德规范》以来, 关于教 师伦理的教育伦理学 (去掉这几个字) 研究更是层出不穷。 [6]教学伦理研究和教师伦理研究, 或者是教师职业道德建 设之间的界限一直比较模糊, 再加上教学伦理从一开始就 比较重视教师的作用, 使得研究者极易因袭这一传统, 在 开展教学伦理研究时更多着眼于教师一方。

虽然教学伦理研究中偏重教师“教”的方面事出有因, 但这并不表示现有的研究结构就是合理的, 对于学习伦理 的遗忘, 不但造成了理论上的空白, 同时也对教学实践造
成了一定的负面影响。因为教学伦理的问题上不仅体现在 教师方面, 也体现在学生方面, 如有部分学生的学习责任 感不强, 学习动力不足, 自我意识过于浓厚, 缺乏对他人 的同情心、关心, 同学之间缺乏交流等, [7]这些学习伦理 的问题同样是教学伦理研究不能回避的重要内容。从系统 论的角度上看, 若想切实提升教学的伦理性, 单从教师一 方着手显然是不够的, 也是不妥的, 因为教师的“教”最终 还是要落实到学生的“学”上, 外因须得借助内因才能发挥 作用, 如果只加强教师“教”的伦理, 而㘣顾学生“学”的伦 理, 学生不买教师的账, 依旧我行我素, 教学伦理也无法 获得切实提升。另外, 单方面强调教师“教”的伦理, 把教 学中的伦理偏差全部归结为教师的责任, 还会造成教师独 负其荷的局面, 加剧其对待教学的消极情绪, 不仅无益于 教学伦理问题的解决, 反而会给教学整体带来负面影响。 由此可见, 只关注教师一方的伦理建设可谓隐忧重重, 必 须通过加强学习伦理研究加以补救。

\section{2. 凸显以规约为基调的规范伦理, 冷落其他多元的伦 理审视}

在研究取向上, 当前教学伦理研究大多遵循规范伦理 学的逻辑, 而对美德伦理、关怀伦理等其他研究视角则少 有问津。所谓规范伦理就是“以原则为基础或以责任为基 础来评判行为的正当、对错与否, 实际上也就是基于行为 或责任的善或恶来讨论道德的善或恶”的一门学问。[8]教 学规范伦理由人的外在行为出发, 勾勒了人们在教学生活 领域中应当遵守的一套道德准则, 强调教师和学生双方需 要履行的义务、承担的责任等, 更多带有底线伦理的意味。 规范伦理之所以能够成为教学伦理研究的主导视角, 主要 还是受到伦理学研究发展脉络的影响, 自从伦理学成为一 门独立的学科, 直至19世纪末元伦理学正式登上西方伦理 学的舞台以来, 规范伦理学都是作为传统伦理学的代名词 而存在。“赫拉克利特面对不公正的生活现象而提出“公正” 问题实际上就是一个伦理问题。而后苏格拉底, 柏拉图的 ‘四德目'理论以及亚里士多德创建伦理学, 都是以规范伦 理为基本内容的。”[9]时至今日, 在经历了一次复归之后, 规范伦理学的主导地位仍难以撼动。另外, 由于规范伦理 学主要着眼于人的外显行为, 具有较强的操作性, 且随着 民主政治的进步以及教育的不断普及, 人们对于教育公平 与正义的关注与日俱增, 也使其在教学领域备受青睐。

诚然, 伦理规范在约束人的行为方面具有难以比拟的 优势, 而“人类发展的未完善性, 人性的不完美性, 以及 教学的社会性, 客观上都需要适度伦理规范的约束”, [10] 但这并不意味着规范伦理就是自洽无缺的, 尤其是在教育 这个关乎人之生命成长的领域, 现有规范是否合理, 能否 引导教师和学生达致较高的价值境界, 都是值得人们反思 的。从实践上看, “现代以来的教学现实表明, 带有令行 禁止味道的各种教学规范并没有实际上帮助师生体会教 学的本真内涵, 而是恰恰相反, 似乎规范越多, 大家的反 感情绪也越强烈。”[11]教学本该是教师倾注热情的事业, 但太多的条条框框, 却难免使人压抑, 那些张贴在教室墙 壁上的行为守则总给人一种冰冷的疏离感, 学生的学习也 受到各种规则的裹挟, 在这样的环境下, 教师教的乐趣和 
学生学的兴致都遭受了一定程度的削弱。教学是一项十分 复杂的活动, 规范是必须的, 但并非所有问题均可依靠原 则妥善解决。比如法国教育社会学家的调查就表明, 不同 学业成就的学生对教师的关怀需要明显不同。学业成绩较 差的学生渴望得到教师积极的态度, 从中获得平等感和信 心, 从而间接地有益于提高学业, 而学业优秀的学生更看 重教师进一步促进自己学业成长的能力和耐心，[12]这些 细微的差别必须以一颗灵动的关怀之心才能体悟, 划一性 的规范则是无法注解的, 而这也提示我们在规范伦理之外, 还需要加入其他视角对教学伦理问题加以审视, 为教学活 动注入更多温情与活力。

\section{3. 固本拓边: 教学伦理研究的战略性调整}

\section{1. 回归教学, 加强学习伦理研究}

教学伦理是关于教学的伦理研究, 其开展理应围绕着 教学的本质进行, 而教学是教师“教”和学生“学”的统一活 动, 在此基础上, 教学伦理也应该是“具有职业身份的教 师履行授业传道的社会责任, 满足学生的求知需求的同时, 以学生为身份的一方也同样履行责任, 双方通力协作, 最 终达到尊师爱生、教学相长的和谐境界。”[13]而在当前只 专注教师“教”的伦理, 忽视学生“学”的伦理之现实困境下, 我们首先要做的就是进一步加强学习伦理的研究。其实, 当前也有一些学习伦理研究的成果, 但显然存在着两大问 题, 一是研究的零散性, 比如, 在我国, 先师孔子就曾对 学生学习提出了学而不厌, 学思结合的要求, 韩愈则提倡 培养刻苦勤学的学习品德, 告诫学生“业精于勤, 荒于嬉”, 当代教育家蔡元培也非常注重学生学习兴趣的培养和学 习责任感的激发, 并详细指出作为学生一要孜孜求学, 二 要砥砥德行, 三要尊师爱友, [14]还认为整顿北大的第一 要务就是改革学生的观念。当前的教学实践实际上也有一 些蕴含着学习伦理的规范要求, 比如中小学生守则等。不 难看出, 无论是古代学习伦理的研究传统, 还是当下对学 生学习的行为规约, 都是一些零散的提法, 尚未进入到教 学伦理领域中, 进行较为系统、全面的研究。

二是研究的片面性, 当前的学习伦理研究强调的多为 学生所应恪守的学习道德、遵循的学习纪律和承担的学习 责任, 这只是学习伦理的一个方面, 学生的学习自由同样 是研究的应有之义。“自律是主体性的表现, 是人出于理 性而为自己立法并出于意志而自愿奉行之”, [15]道德强调 和追求自由, 其实质是主体对客体的认识、选择、改造和 超越, 是主体性的充分表现。恩格斯说, “如果不谈谈所 谓自由意志、人的责任、必然和自由的关系等问题, 就不 能很好讨论道德和法的问题。”[16]因此, 学习自由可以说 是学习伦理的重要组成部分, 有些研究者可能会认为强调 学习自由会给教学带来混乱, 而刻意回避之, 这是大可不 必的, 实质上真正的自由被天然地赋予了责任, 哈耶克曾 指出“自由不仅意味着个人拥有选择的机会并承受选择的 重负, 而且还意味着他必须承担其行动的后果, 接受对其 行动的赞扬或谴责。”[17]可见, 学习自由不仅不会戕害教 学秩序, 还是促成学生成长的重要条件。
除此以外, 在研究教学伦理时, 我们还需要注意教师 “教”的伦理和学生“学”的伦理之融通。“教学概念的核心精 神即在“教学”中“教”与“学”是统一的, 只有理论研究或实践 中着力有所侧重的情况, 不存在“分”或“合”的问题, 一旦 真的“分开”, 则教学就消失, 就不复存在。”[18]比如, 有 研究关注到了学生学习责任的重要性, 但却仅仅探讨了学 习责任与学习负担的关系, [19]只是就学习而论学习, 并 没有体现教学活动的特殊性。因而, 在分别加强“教”的伦 理和“学”的伦理的基础上, 还要致力于二者的融合, 关注 “教”和“学”的互动, 比如有研究者就基于师生关系, 提出 了“教学道德调节”的概念, 论述了师生共同遵守的道德原 则和规范。这些原则和规范有“爱书和思考”、“练习和创 造”、“通心与共慰”。[20]教学是一个系统, 为了研究的方 便, 我们可以将其中某个要素剥离出来看待, 但“拿得出” 容易, 更要“放得回”, 否则教学伦理研究便会被肢解开来, 丧失了对其研究的真正意义。

\section{2. 多维审视, 提升理论指导的适切性}

教学是一项十分复杂的活动, 充满了不确定性, 尽管 规范伦理在约束人的行为方面很有必要, 但并不充分。因 为任何一种理论都不过是以某一特殊视角来解释伦理生 活, 无法涵盖其中所有问题。上文已经提到规范伦理更多 强调义务、规则或后果, 主要聚焦于善恶、正义和公平问 题的研究, 带有较强的纲性意味, 对于公共生活的利益调 节和行为规范十分有益。但教学中的很多问题不是按照规 则就能解决的, 而是需要教师的伦理智慧和学生的伦理自 觉。比如, 教师已经完成了规定的教学任务, 但某些学生 还是没有掌握, 此时如果仅遵从规范伦理的条目, 教师就 可以心安理得地离开学校。对于学生而言, 也是如此, 如 果同学有不明白的问题向自己寻求帮助, 到底要不要牺牲 游戏的时间来完成这件看似分外的事情, 这些都不属于规 范伦理的研究范畴, 而是需要美德伦理的指引。此外, 教 学也不仅关乎专业, 更需要投注热情, 有一些教师虽然能 够上好课, 但对学生的关心却很不到位, 或者采取的方式 不得当, 这就需要关怀伦理学加以补充。概而言之, 教学 中的伦理活动不仅要“讲理”, 同时也要“谈情”, 二者缺一 不可, 道德不仅是遵守, 更需要内在的力量和衷心的关切, 在教学的程序性问题和公共事务中, 规范显然处在更为突 出的地位, 而在教学的主体品行和私人交往中, 则更需要 美德和关怀的力量显现。正如有研究者指出的那样, “各 种不同的理论形态, 在通达幸福之路上, 都有各自的位置”, [21]教学伦理研究也是如此, 需要各种视角的会同交融, 以便为教学伦理发展提供全面的支持。

除此以外, 在引入各种教学伦理研究视角的过程中还 要关注教学的特有属性, 因为无论是哪一种伦理范式, 都 是对于一般伦理问题的研究, 并不能完全适应于教学领域, 只有经过一定改造, 方可使用。仅以关怀伦理为例, 教师 对于学生的教学关怀, 应在日常关怀真情性的基础之上, 更加强调关怀的责任性和科学性。日常生活中人们相互的 嘘寒问暖, 只要有一颗真挚的心, 一片深切的情就足够了, 但教学中的关怀却要具备一定的引领作用, 在反对功利主 义倾向的同时, 也要考虑实际的教育效果。这一方面对教 
师的责任和素质提出了要求, 比如教师始终有责任将自己 真实的想法告知学生, 并帮助学生在充分知情的情况下尽 可能做出正确的选择。[22]而且教师的关怀还要建基于对 教学的科学认识之上, 以教育学、心理学的相关知识为指 导, 结合学生个体情况施以关怀, 明确关怀的内容并选择 适当的关怀方式。像因材施教这一理念就体现了教师对所 有学生的尊重, 蕴含着教学的人性化关怀, [23]但要想真 正实现因材施教, 仅靠教师对学生的教育爱是不够的, 还 需要教学理论的指导。另一方面, 也对学生的成长与发展 提出了期待, 比如以关怀伦理统领教学活动时, 就不能仅 仅满足于让学生体会到教师的关爱, 还要不断提升学生感 受关爱、回应关爱和给予关爱的意愿和能力, [24] 以确保 教学伦理活动能充分体现其教育价值。总之, 无论是以多 元视角审视教学伦理问题, 还是在研究中赋予各种伦理视 角以教学意蕴, 都是为了提升教学伦理的适切性, 更好地 为教师发展、学生成长服务。

\section{4. 结论}

当前的教学伦理研究存在较为明显的结构性缺失。首 先, 在教学活动主体方面, 偏指教师一方, 主要聚焦于教 师的职业伦理, 对于学生的学习伦理则鲜有关注, 忽视了 学生在伦理活动中的主体地位。这虽在很大程度上源于师 生关系的特殊要求, 但却枉顾了教学活动的双边性, 一方 面会造成教师独负其荷, 另一方面也不利于学生个体责任 感的培养。为此, 需要回归“教学”本质, 加强学习伦理的 专门研究, 挖掘我国古代优秀的学习文化传统, 结合近代 西方学习自由的相关思想, 对学习伦理进行系统、全面的 梳理, 并将其置于教学活动这一语境中加以思考, 注意研 究过程中“教”与“学”的统一。

其次, 在教学伦理研究视角方面, 仍以规范伦理为主 流, 强调规则与理性的价值, 没有很好地与其他研究取向 进行有益结合。教学作为一种特殊的社会活动, 其终极目 的并不在于使学生牢记和遵守规范, 而是要发展人, 成就 人, 这就决定其不能简单沿袭一般伦理学研究的传统格局。 而且现代以来的教学现实也表明, 带有令行禁止味道的各 种教学规范并没有实际上帮助师生体会教学的本真内涵, 反而容易招致教学主体的反感情绪。可见, 仅以规范伦理 视角对教学伦理问题加以审视已无法满足当前教学伦理 的发展, 这就需要加入美德伦理学、关怀伦理学等多重视 角, 以提升问题解决的适切性。此外, 在研究过程中, 还 应当对这些伦理学理论加以改造, 以突出其教育价值与成 人使命。

\section{参考文献}

[1] 汪明, 张睦楚. 批判与期盼: 关于教学活动性质的理性思 考与深层追问 [J]. 湖北社会科学, 2015（10）：157-162。

[2] 汪明, 张睦楚. 对开展教学伦理学研究反对之声的回应与 批判 $[\mathrm{J}]$. 中国教育学刊, 2015 (8) : 4-8+72。
[3] 王本陆, 王婵.简议教学伦理研究中的学生主体问题[J].教育 学报, 2017（5）：33-37。

[4] 王策三. 教学论稿 (第二版) [M]. 北京: 人民教育出版社, 2005: 87-88。

[5] 侯丽娜.中小学合作学习中的伦理失衡及其矫正策略[J].教 学与管理, 2017 (14)：1-3。

[6] 王凯.教学伦理研究的现状与问题[J]. 全球教育展望, 2008 (1) : 50-53。

[7] 胡斌武. 教学伦理探究[M].成都: 四川教育出版社, 2005: 1。

[8] 刘丙元. 从规范到德性: 当代道德教育哲学的本真回归 $[\mathrm{J}]$. 理论导刊, 2010（1）：34-37。

[9] 唐代兴.变革与图新: 发展规范伦理学的当代思路 [J].道德与 文明，2013（1）：110-115。

[10] 刘万海.关于教学道德性的原点审思 [J].全球教育展望, 2007 (1) : 31-39。

[11] 刘万海, 李倩.试论当代教学伦理研究路向的转换[J]. 全球教 育展望, 2008（2）：14-18。

[12] Noddings, N. Caring: A Femine Approach to Ethics and Moral Educational.1984: 178-179.

[13] 曾钊新. 试论教学中的道德调节[C]. 北京: 人民教育出版社, 1988: 132。

[14] 钱焕琦主编.中国教育伦理思想发展史[M].北京：改革出版 社, 1998: 126-134。

[15] 王本陆.教育崇善论[M].广州: 广东教育出版社, 2001:319。

[16] 马克思恩格斯选集 (第1卷) [M]. 北京: 人民出版社, 1972: 153-154。

[17] 哈耶克. 自由秩序原理[M]. 邓来正, 译. 北京: 三联书店, 1988: 102 。

[18] 丛立新. 教学概念的形成及意义[J]. 北京师范大学学报 (社 会科学版)，2007（5）：5-12。

[19] 余文婧.论学习的责任[J].江西师范大学学报 (哲学社会科学 版），2016（6）：167-174。

[20] 篗葆奎主编.教育学文集・教学 (上册) [M].北京：人民教育 出版社, 1988：132-145。

[21] 高国希.道德理论形态: 视角与会通[J]. 哲学动态, 2007(8): 25-29。

[22] 侯晶晶, 朱小曼. 诺丁斯以关怀为核心的道德教育理论及其 启示 [J].教育研究，2004（3）：36-43。

[23] 欧阳超.教学伦理学 [M].成都: 四川大学出版社, 2008: 149。

[24] 张崇, 付梅.回应关怀: 关怀以教育的“软肋”[J].现代中小学 教育，2014（8）：39-42。 